\title{
Optimization of Mobile IPv6 Handover Performance Using E-HCF Method*
}

\author{
Guozhi.Wei ${ }^{1}$, Anne.Wei ${ }^{1}$, Ke.Xu ${ }^{2}$, and Gerard.Dupeyrat ${ }^{1}$ \\ ${ }^{1}$ University of Paris XII 61, Avenue du General de Gaulle 94010 Créteil France \\ ${ }^{2}$ Tsinghua University 100084 Beijing China \\ \{guozhi.wei, wei,g.dupeyrat\} @univ-paris12.fr, \\ xuke@mail.tsinghua.edu.cn
}

\begin{abstract}
Mobile IPv6 permits the Mobile Node (MN) to maintain the continuous connectivity to the Internet when it moves from one access router to another. Due to both link switching delay and IPv6 protocol operation during the handover process, packets destined to the $\mathrm{MN}$ can be delayed or lost. This paper proposes a solution to improve the Mobile IPv6 handover performance over wireless network by introducing a new entity - Extension Handover Control Function (E-HCF). E-HCF could send the decisive control message to $\mathrm{MN}$ to accelerate the handover process and manage the traffic belonging to $\mathrm{MN}$ to reduce the packet loss. With a comparison between Mobile IPv6 and our E-HCF solution, we show that our solution allows us providing low-latency and low packet loss for real-time services during the handover.
\end{abstract}

Keywords: Mobile IPv6, Handover, E-HCF function, WLAN.

\section{Introduction}

The need of keeping connection with Internet in everywhere and at every time is more and more demanded in recently years. However, the continuous Internet connectivity and the correct routing of packets could not be guaranteed when users change their access point to the Internet. To resolve these problems, the protocols Mobile IPv4 (MIPv4) [1] and Mobile IPv6 (MIPv6) [2] are respectively proposed by the Internet Engineering Task Force (IETF). The major difference between MIPv4 and MIPv6 is that Foreign Agent (FA) is elided in the latter; moreover Mobile Node (MN), its Home Agent (HA) and its Correspondence Node (CN) must all support the IPv6 Protocol. The MIPv6 operations involve movement detection, router discovery, Care of Address (CoA) configuration, Duplication Address Detection, and Binding update. Although MIPv6 is proposed in the interest of improving the handover performance, the latency and packet loss are always two main problems, which affect the real-time application employing for mobile user.

Along with the widely implantation of Wireless LAN (WLAN) [3], the user of WLAN would like to have the more mobility. To meet this demand, the MIPv6 is needed to be better improved over the WLAN. While the infrastructure networks are already installed, such as Access Router (AR), Access Point (AP), they are hardly

\footnotetext{
* This work was supported in part by the international project PRA-SI under Grant SI04-03.
} 
updated or modified. Under this background, we introduce a new entity - Extension Handover Control Function (E-HCF) in Mobile IPv6 over WLAN to improve the handover performance without changing the existing infrastructures. As the probe phase (MN launches the scanning process to find the available AP in WLAN.) [3] and Detection Address Duplication (DAD) process are the main negative influences on handover latency, E-HCF aims to improve the handover performance by reducing their effects. Moreover, to reduce the packet loss, E-HCF could buffer, and then redirect the traffic according to the MN's need during the handover process to reduce the packet loss.

The remainder of the paper is organized as follows: Section 2 presents background and related work of Mobile IP. Section 3 presents our Extension Handover Control Function (E-HCF) architecture and the detailed protocol operation associated. Section 4 deals with the performance of E-HCF handover in term of handover latency and packet loss. The numerical results show that E-HCF handover procedure reduces significantly the latency and packet loss regarding the Mobile IPv6. Finally, conclusion and future work are mentioned in section 5 .

\section{Background and Related Works}

Actually, the main proposals accepted by IETF are Hierarchical Mobile IPv6 (HMIPv6) [4] and Fast Handover for MIPv6 (FMIPv6) [5].

HMIPv6 introduces an entity - Mobility Anchor Point (MAP) who acts somewhat like a local HA for the visiting MN. HMIPv6 classes MN mobility into micromobility (within the same MAP domain or intra-MAP) and macro-mobility (between the two MAP domains or inter-MAP). When intra-MAP handover occurs, MN only needs to register its new CoA with its serving MAP, therefore, HMIPv6 could limit the amount of signal required outside the MAP's domain, decrease the signal load and the signal transmission delay, and consequently, the handover performance could be improved. While HMIPv6 introduces the additional delay for establishing the bidirectional tunnel between MAP and MN, generates the more signal load for InterMAP handover. Furthermore, it is difficult to determine an adaptive MAP domain size for different MN's mobility pattern [6] [7].

FMIPv6 tries to reduce handover delay by providing fast IP connectivity as soon as $\mathrm{MN}$ attaches to a new subnet. To realize this aim, MN must launch the passive or active scanning process to discover the available AP [8]. According to the probe results, AR provides $\mathrm{MN}$ with the corresponding subnet prefix information, and then MN could generate a New CoA when it is still connected to its current subnet. To minimize packets loss, a bi-directional tunnel is set up between Previous AR and New AR. Utilizing this tunnel, PAR forwards the packets destined to MN's Previous CoA to its New CoA, MN could also continue to send packets to $\mathrm{CN}$ through PAR. Such tunnel remains active until $\mathrm{MN}$ completes Binding Update with its CNs. However, there are two mains shortcomings in the FMIPv6 protocol. Firstly, MN couldn't receive or send the data during the probe phase, while it lasts minimum $350 \mathrm{~ms}$ [9], furthermore, MN must spend time to re-switch the channel and re-associate with its Previous AP to exchange the messages with PAR; Secondly, the DAD process could 
not be completely avoided if MN's New CoA isn't validated by the New AR before MN disconnects with its previous AR.

Besides the main proposals, some approaches are proposed to providing lossless handover and minimizing the handover delay ([10], [11], [12]). IEEE 802.11f standard, known as Inter-Access Point Protocol (IAPP), has been proposed in 2003[10]. IAPP specifies the information to be exchanged between APs to support MN handover. But this standard has been withdrawn on February 2006 by the IEEE 802 Executive Committee. In [11], a Pre-Handover Signaling (PHS) protocol is proposed to support the triggering of a predictive handover and to allow the network to achieve accurate handover decisions considering different constraints, such as Quality-ofService (QoS), the user's profile and the MN service requirements. In [12], this paper proposes to use a new entity - Handover Control Function (HCF) to pre-decide MN's new CoA, consequently, MN could send Binding Update message when it still connects with its previous AP. However HCF could not avoid absolutely the IP collision and the inter-HCF handover is not discussed. Therefore, in this paper, we use E-HCF entity to reduce the layer 2 scanning delay, avoid the duplication of IP address, and support the inter-E-HCF handover.

\section{Extension Handover Control Function (E-HCF) for Mobile IPv6}

\subsection{E-HCF Overview}

We introduce a local intelligent entity, called E-HCF, which is able to control the ARs, APs and MNs of its domain. The architecture of E-HCF is shown in Fig 1.

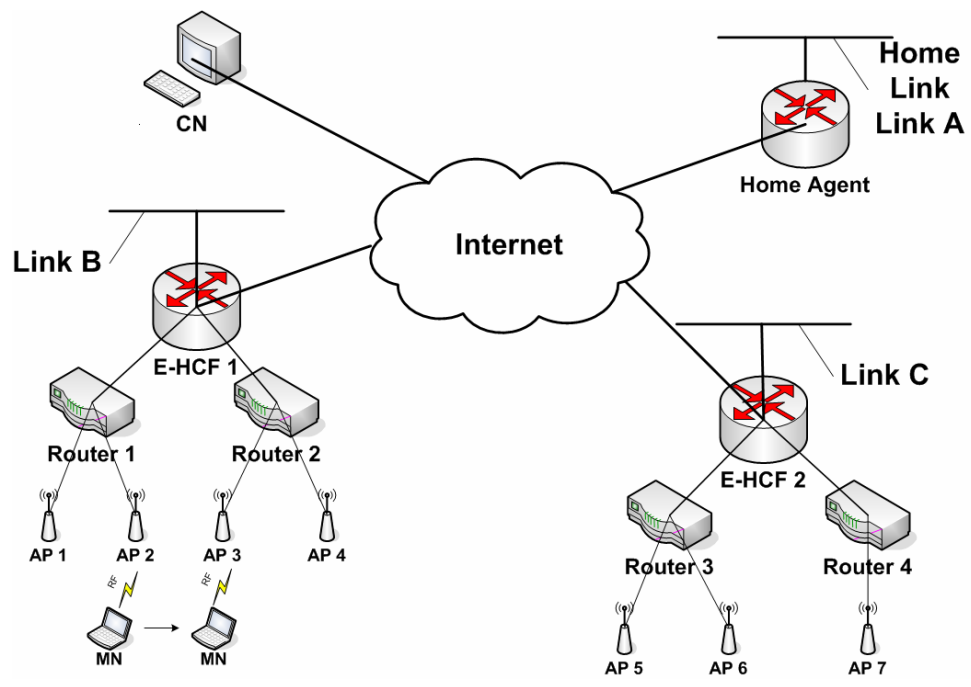

Fig. 1. Architecture of Extension Handover Control Function (E-HCF)

In order to improve the MIPv6 handover performance over WLAN, we endow E-HCF with the new functions: E-HCF could provide a list of the AP as the available 
AP and a corresponding IP address as the new CoA for MN. In this way, MN needn't launch the scanning process to discovery the available AP and the Detection Address Duplication (DAD) process in the new subnet.

E-HCF could provide beforehand a list of the APs which MN could potentially connect to in accordance with the MN's location (in other words, based on the location of MN's current AP). Moreover, E-HCF defines the priority of the available APs according to the AP's charge, the network balance, the MN's trajectory, etc. If necessary, E-HCF could enquire its contiguous E-HCFs to offer their APs and relative information via the Int-E-HCFReq and Int-E-HCFRep messages [13].

E-HCF reserves a pool of IP address to form a list of available CoA for MN. Simultaneously, E-HCF generates and updates periodically a special IP address list - a list of IP address used by the nodes of its domains. By comparing these two lists, EHCF could find the potential address collision in its domain. If the reserved IP address were used by a node, E-HCF would either withdraw this IP address from the fist list or demand the node to change its IP address. In this way, we could assure that once EHCF distributes a reserved IP address to MN, MN could use this IP address directly in the new subnet.

We add six new messages into Mobile IPv6 protocol. E-HCF could use these messages to exchange both the intern (local) information with its ARs/MNs and the extern information with another E-HCF. These messages are the following: MN Request (MNReq), E-HCF Reply (E-HCFRep), Inter-E-HCF Request (Int-E-HCFReq), Inter-EHCF Reply (Int-E-HCFRep), Connection Established Information (CEInf) and Handover Finished Confirmation (HFCon) messages. Due to the limited pages, the formats of these messages won't be given at here, please read our technique report [13].

\subsection{E-HCF Procedure}

In this sector, we give the detail handover procedure to the well understand of our proposition. As shown in Figure 2, E-HCF procedure is detailed as follows:

- MN moves in the network, once the threshold of received signal strength is overstepped, it sends a MNReq Message to E-HCF immediately to request the network information, such as the available APs, its BSSID, its channel, the prefix of the corresponding ARs, etc.

- E-HCF replies to MN with the E-HCFRep message. MN could obtain the needed information, and the prospective corresponding CoA from this message.

- Once MN receives the E-HCFRep message, MN begins to associate with the first AP of the list. If MN could establish the connection with the first AP, MN uses the proposed CoA to send the Binding Update to its HA, its CNs, and sends the CEInf message to E-HCF to notify its attachment. Otherwise, it does the same in order to connect to the better AP.

- To avoid packets loss, E-HCF commences buffering the traffic destined to MN's previous CoA when it receives the MNReq message. Once it receives the CEInf message, it sends the buffered packets to MN's new CoA. E-HCF sends HFCon message to MN when it couldn't receive the traffic destined to MN's previous CoA any more. 


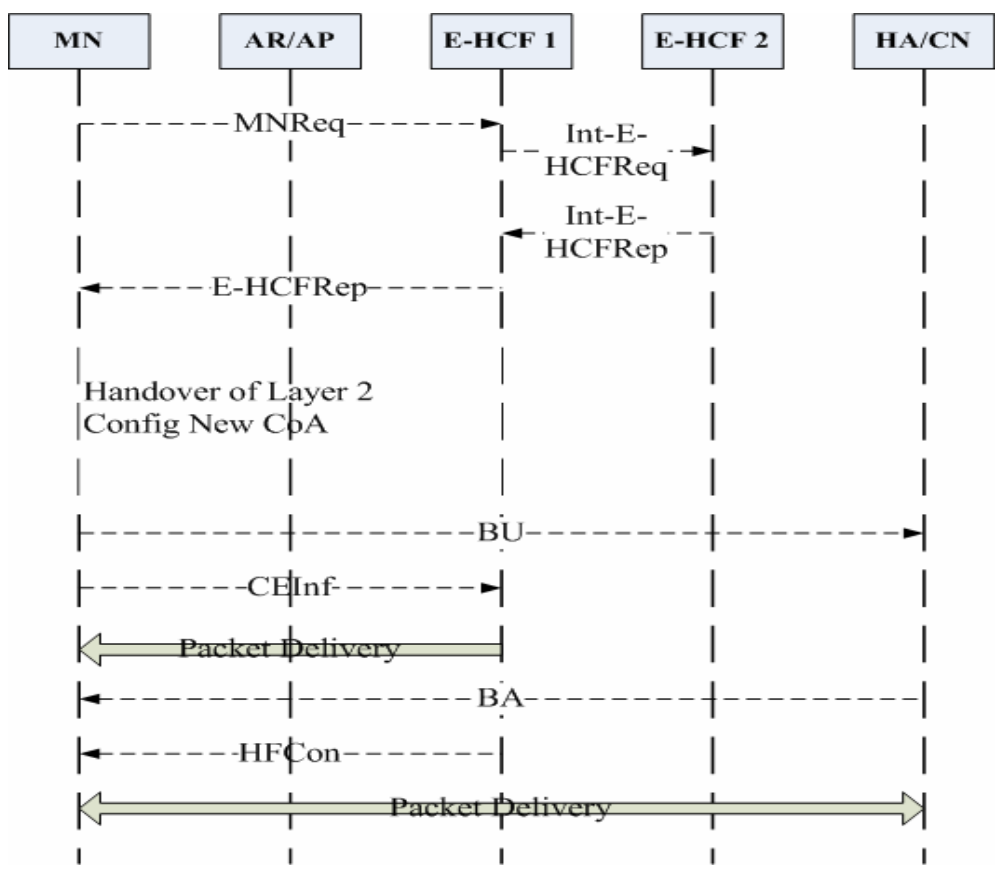

Fig. 2. E-HCF procedure

Recall that Int-E-HCFReq/Int-E-HCFRep messages are exchanged between the two E-HCFs for MN's inter-E-HCF handover. Each E-HCF maintains and updates its proper network information.

\section{E-HCF Performance Estimation}

The MIPv6 handover over WLAN consists of Link Layer handover and Network Layer handover. Link Layer handover includes Probe phase, Authentication phase, and Re-association phase. Network Layer handover includes Router Discovery phase, DAD phase and Binding Update phase. Displayed in Figure 3, the handover latency

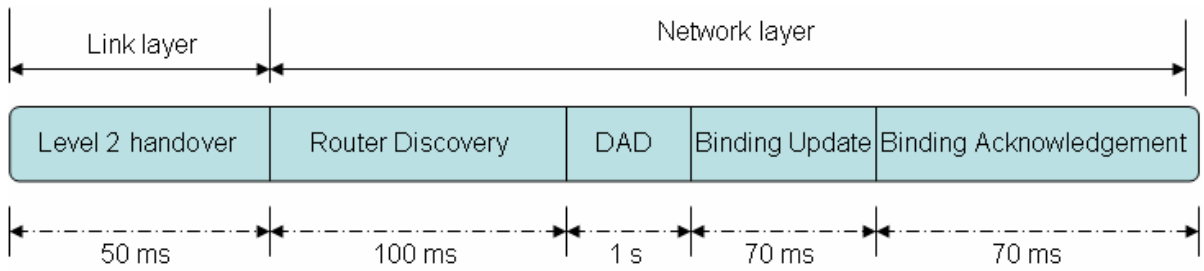

Fig. 3. Standard MIPv6 Latency 
could be estimated to minimum $1290 \mathrm{~ms}$ [14] without including probe phase delay (between $350 \mathrm{~ms}$ and $500 \mathrm{~ms}$ ). If we analyze each phase during handover process, we can observe that the probe phase and DAD phase delay costs $350 \mathrm{~ms}$ and $1000 \mathrm{~ms}$ respectively. In another word, probe phase and DAD phase are the key argument for the handover latency. In the following sections, we try to prove our proposition by mathematic analysis and compare E-HCF with MIPv6.

Before the detail latency analysis, we give the following notations:

$\begin{array}{ll}\mathrm{L}_{\mathrm{MIPv} 6} & \text { Total handover latency with MIPv6 } \\ \mathrm{L}_{\mathrm{E}-\mathrm{HCF}} & \text { Total handover latency with E-HCF. } \\ \mathrm{L}_{\text {Prob }} & \text { Latency that MN scans all neighboring AP to find the available AP } \\ \mathrm{L}_{\text {Authentication }} & \text { Latency for authentication phase of Link Layer handover. } \\ \mathrm{L}_{\text {Association }} & \text { Latency for association phase of Link Layer handover. } \\ \mathrm{L}_{\text {Router Discovery }} & \text { Latency for Router Discovery phase of Network Layer handover } \\ \mathrm{L}_{\text {DAD }} & \text { Latency for DAD phase of Network Layer handover } \\ \mathrm{L}_{\text {BU/BA }} & \text { Latency for Binding Update phase of Network Layer handover } \\ \mathrm{L}_{\text {MNReq }} & \text { Latency that MN sends a MNReq message to its E-HCF. } \\ \mathrm{L}_{\mathrm{E}-\mathrm{HCFRep}} & \text { Latency that E-HCF sends E-HCFRep message to MN, including } \\ & \text { sometimes the delay for the Int-E-HCFReq message/Int-E-HCFRep } \\ & \text { message exchange. } \\ \mathrm{L}_{\mathrm{CEInf}} & \text { Latency that MN sends CEInf message to E-HCF } \\ \mathrm{L}_{\mathrm{HFCon}} & \text { Latency that E-HCF sends HFCon message to MN }\end{array}$

In according to the procesus illustrated in above section.

The total MIPv6 handover latency $\mathrm{L}_{\mathrm{MIPv} 6}$ is:

$\mathrm{L}_{\text {MIPv6 }}=\mathrm{L}_{\text {Prob }}+\mathrm{L}_{\text {Authentication }}+\mathrm{L}_{\text {Association }}+\mathrm{L}_{\text {Router Discovery }}+\mathrm{L}_{\mathrm{DAD}}+\mathrm{L}_{\mathrm{BU} / \mathrm{BA}}$

The total E-HCF handover latency $\mathrm{L}_{\mathrm{HCF}}$ is:

$\mathrm{L}_{\mathrm{HCF}}=\mathrm{L}_{\mathrm{MNReq}}+\mathrm{L}_{\mathrm{E}-\mathrm{HCFRep}}+\mathrm{L}_{\text {Authentication }}+\mathrm{L}_{\text {Association }}+\mathrm{L}_{\mathrm{BU} / \mathrm{BA}}+\mathrm{L}_{\text {CEInf }}+\mathrm{L}_{\mathrm{HFCon}}$

When we compare our E-HCF approach with MIPv6, we find that the key negative arguments of handover latency (such as $\mathrm{L}_{\mathrm{Prob}}, \mathrm{L}_{\mathrm{Router}}$ Discovery, $\mathrm{L}_{\mathrm{DAD}}$ ) could be eliminated from our E-HCF proposition. Although the latency for messages exchange is introduced, latency for messages exchange would not be important.

In term of the packet loss, E-HCF commences buffering the traffic destined to MN's previous CoA when it receives the MNReq message. Once it receives the CEInf message, it sends the buffered packets to MN's new CoA. If we consider that the size of E-HCF buffer is sufficient for buffering all packets destined to MN's previous CoA, which arrived before E-HCF receives CEInf message and begins to sends the packets to MN's new CoA, the packet loss gets its value to zero. Moreover, according to our E-HCF approach, the duration between the time that E-HCF commences buffering the packets and the time that E-HCF receives CEInf message and begins to sends the packets to MN's new CoA is quite short. 


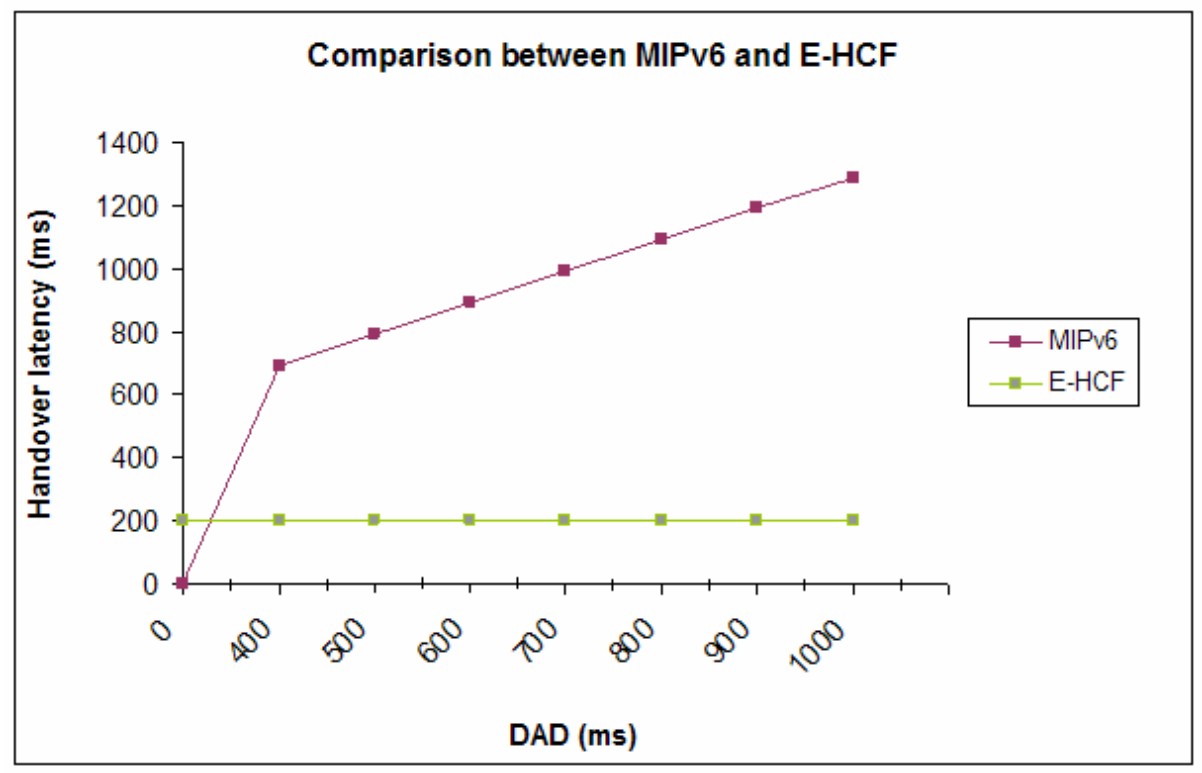

Fig. 4. E-HCF handover latency comparison in function of DAD

\section{Conclusions}

This paper proposes a new entity - E-HCF to improve the handover performance in Mobile IPv6 over WLAN. According to our analysis, both the handover delay and the packet loss could be significantly decreased. Moreover, E-HCF could enhance the AP's security, because AP needn't broadcast its SSID any more to permit MN to discover itself. Furthermore, E-HCF permits MN to connect to the authorized AP by adding the corresponding security parameters (such as WEP/WPA) into the encrypted E-HCFRep messages.

While how to better choose the available AP and define the AP's priority according to MN's mobility pattern is still a challenge for us. What's more, we need guarantee the QoS service for diverse MNs which have the different traffic type, the different service demand, and the different priority. Our current work focuses on resolving above problems and using the OPNET (Optimized Network Engineering Tool, a network simulator) [15] to prove our proposition. The wireless handover in MIPv6 will be studied in the future.

\section{References}

[1] C. Perkins, "IP Mobility support for IPv4", RFC 3220, IETF, January 2002.

[2] D.Johnson, C. Perkins, and J.Arkko, "Mobility Support in IPv6", RFC 3775, June 2004.

[3] B. O'Hara, A. Petrick, "IEEE 802.11 Handbook- A Designer's Companion", IEEE Press.

[4] H.Soliman, C.Castelluccia, K.Malki, and L.Bellier, "Hierarchical Mobile IPv6 mobility management (HMIPv6)", RFC 4140, August 2005. 
[5] R. Koodli, Ed. "Fast Handovers for Mobile IPv6", RFC 4068, July 2005.

[6] Bok-Deok Shin, Kyung-Jae Ha, "An Improvement of Handoff Latency by Virtual MAPs for HMIPv6", in Proceedings of the IEEE CIT 2006 p.78

[7] Sangheon Pack, Minji Nam, and Yanghee Choi, "Design and Analysis of Optimal MultiLevel Hierarchical Mobile IPv6 Networks," Springer (Kluwer) Wireless Personal Communications (WIRE), Vol. 36, No. 2, January 2006.

[8] Mishra, M.Shin, and W.Arbaugh, "An Empirical Analysis of the IEEE 802.11 MAC layer Handoff Process", ACM Computer Communications Review, vol. 33, no. 2, Apr. 2003.

[9] Ishwar Ramani, Stefan Savage, " SyncScan: Practical Fast Handoff for 802.11 Infrastructure Networks", Proceedings of the IEEE Infocom Conference, Miami, FL, March 2005

[10] "IEEE 802.11f: Recommended Practice for Multi-Vender Access Point Interoperability via an Inter-Access Point Protocol Access Distribution Systems Supporting IEEE 802.11 Operation”, IEEE Standard 802.11, Jan. 2003

[11] H.Chaouchi, P.Antunes, "Pre-handover signaling for QoS aware mobility management," international journal of network management 14, pp.367-374, 2004;

[12] G. Z. Wei, A. Wei, K. Xu and H. Deng, "Handover Control Function Based Handover for Mobile IPv6," In Proceedings of ICCS 2006, May 2006.

[13] Guozhi WEI, "E-HCF function and messages formats", research report 2006-145, University of Paris XII -Val de Marne

[14] Wei Kuang Lai and Jung Chia Chiu, "Improving Handoff Performance in Wireless Overlay Networks by Switching Between Two-Layer IPv6 and One-Layer IPv6 Addressing," IEEE Journal on Selected Areas in Communication vol. 23, No.11, November 2005.

[15] C. Zhu, O. W. W. Yang, J. Aweya, M. Oullette, and D. Y. Montuno. "A comparison of active queue management algorithms using the OPNET Modeler." IEEE Communications Magazine, 40(6): 158-167, 2002. 